\title{
Jésuites violents et poissons volants
}

\section{Dominique Descotes}

\section{OpenEdition \\ Journals}

Édition électronique

URL : http://journals.openedition.org/ccibp/282

DOI : $10.4000 /$ ccibp. 282

ISSN : 2493-7460

\section{Éditeur}

Centre international Blaise Pascal

\section{Édition imprimée}

Date de publication : 1 décembre 2012

Pagination : 28-36

ISBN : 978-2-84516-612-7

ISSN : 0249-6674

Référence électronique

Dominique Descotes, " Jésuites violents et poissons volants 》, Courrier du Centre international Blaise Pascal [En ligne], 34 | 2012, mis en ligne le 03 décembre 2015, consulté le 23 avril 2019. URL : http:// journals.openedition.org/ccibp/282 ; DOI : 10.4000/ccibp.282

Ce document a été généré automatiquement le 23 avril 2019

Centre international Blaise Pascal 


\title{
Jésuites violents et poissons volants
}

\author{
Dominique Descotes
}

1 Le fragment Laf. 909, Sel. 451, qui figure à la page 344 du Recueil des originaux des Pensées, s'en prend aux jésuites en ces termes : "Gens sans paroles, sans foi, sans honneur, sans vérité, doubles de cœur, doubles de langue et semblables, comme il vous fut reproché autrefois, à cet animal amphibie de la fable, qui se tenait dans un état ambigu entre les poissons et les oiseaux. " A ma connaissance, cette formule, dont le caractère baroque ne paraît pas caractéristique du style de Pascal, n’a jamais été commentée.

2 Le texte est hétérographe, parmi d'autres notes, qui, elles, sont autographes, et qui traitent de sujets divers.

Figure 1

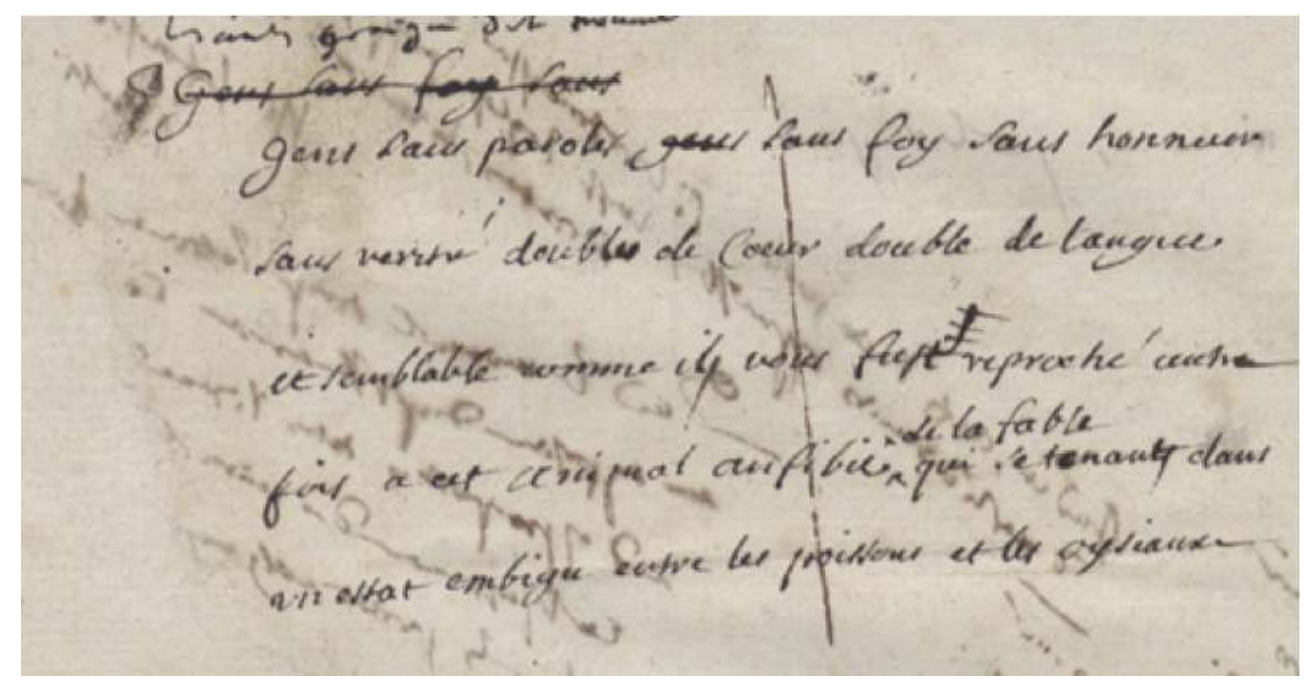

Gens sans foy sans

Gens sans parole, gens-sans foy sans honneur sans vérité doubles de Cœur, double de langue et semblable comme il vous fust reproché autre 
^de la fable

fois a cet animal anfibie ${ }^{\wedge}$ qui se tenait dans

un estat ambigu entre les poissons et les oysiaux

4 Le copiste a commencé par Gens sans foi, il allait continuer, sans doute par sans honneur, et il a tout repris parce qu'il s'est aperçu qu'il avait omis Gens sans parole. L'expression de la fable est une addition signalée par le signe d'insertion ^ ${ }^{\wedge}$, fréquent dans le manuscrit des Pensées. Le texte a été barré verticalement, ce qui, dans les habitudes de Pascal, suppose qu'il a été utilisé. On ne retrouve pourtant cette citation nulle part dans les textes connus.

Le texte est manifestement composé de deux parties. La première consiste en une série d'invectives, dont on peut trouver des échos dans les Provinciales, notamment dans la quinzième et la seizième, qui traitent de la calomnie, de la duplicité des jésuites. Pascal a montré par l'affaire de Benoît Puys que les jésuites changent de discours d'un moment à l'autre, suivant ce qu'ils jugent être leur intérêt: sans foi signifie dans le cas présent incapable de bonne foi, et indigne de confiance. On sait aussi que dès la XIII ${ }^{e}$ Provinciale, § 24, Pascal écrit: «Concluons donc, mes Pères, que puisque votre probabilité rend les bons sentiments de quelques-uns de vos auteurs inutiles à l'Église, et utiles seulement à votre politique, ils ne servent qu'à nous montrer, par leur contrariété, la duplicité de votre cœur, que vous nous avez parfaitement découverte, en nous déclarant d'une part que Vasquez et Suarez sont contraires à l'homicide, et de l'autre, que plusieurs auteurs célèbres sont pour l'homicide, afin d'offrir deux chemins aux hommes, en détruisant la simplicité de l'Esprit de Dieu, qui maudit ceux qui sont doubles de cœur, et qui se préparent deux voies : Vae duplici corde, et ingredienti duabus viis! » Dans son commentaire de l'Ecclésiastique, II, 14, Sacy explique : «Malheur à ceux qui ont deux cœurs, l'un pour Dieu, l'autre pour le monde; malheur à ceux dont les lèvres sont aussi corrompues que le cœur, puisque la langue est nécessairement double si le cœur est double. Marcher par deux voies est lorsque l'on marche selon Dieu en apparence, et selon le monde dans le fond du cœur. »

6 Le binaire, qui exprime la dualité, est souvent considéré comme symbole de duplicité, et par suite de l'hypocrisie. L'hypocrite est donc souvent comparé à des animaux que leur nature situe entre deux milieux. C'est ce que l'on voit par exemple chez Pietro Bongo (Petrus Bungus), chantre et chanoine de Bergame, érudit notamment dans la cabale, mort en 1601. Il est auteur d'un De mystica numerorum significatione, Bergame (1583 et 1584), dont la troisième édition porte le titre de Numerorum mysteria ex abditis plurimarum disciplinarum fontibus hausta, Opus maximarum rerum doctrina et copia refertum. In quo mirus imprimis, idemque perpetuus arithmeticae Pythagoricae cum divinae paginae numeris consensus multiplici ratione probatur (Bergame, 1585). Une édition a été publiée à Paris en 1618, sous le titre Opus maximarum rerum doctrina et copia refertum. In quo mirus imprimis, idemque perpetuus arithmeticae Pythagoricae cum divinae paginae numeris consensus multiplici ratione probatur. On y lit, De binario, p. 79: «Binarius enim hypocritas maxime notat, qui nunc spiritualia sectari videntur, nunc terrenis actionibus et negotiis involvuntur, similes prorsus quibusdam piscibus sive animalibus, quae nunc in aquis, nunc in terris versantur ; inde ab Isidoro appellantur dubia, seu ancipitis naturae, quod non solum natatilia, sed et gressibilia sint per naturam, et utroque elemento participent cujusmodi sunt, phocae, castores, testudines, cocodrili, et hujusmodi, quae communiter sint animalia saeva, vel maligna, et hujusmodi homines dubium videtur utrum sint Dei vel mundi : deceptores tamen sunt et iniqui, de quibus legitur scriptum : omnis hypocrita est et nequam; et alibi dicitur, quia nemo militans Deo implicat se negociis secularibus, ut placeat Deo ${ }^{1}$.» 
7 Ce texte ne saurait être considéré comme une source, car l'idée de fable manque. D'autre part, si les animaux mentionnés chez Bongo, phoques, castors, tortues et crocodiles, sont des amphibies, qui vivent dans deux milieux différents, ils ne sont pas vraiment doubles, ni composés comme l'est l'animal qu'évoque Pascal.

On en trouve des exemples plus pertinents dans Mersenne, La vérité des sciences : à côté des choses inanimées comme « le limon » qui est « comme un lien entre l'eau, et la terre, les vapeurs sont entre l'air et l'eau, les exhalaisons entre l'air et le feu, les comètes, et les autres météores entre le feu et le ciel : l'argile est entre le limon, et les pierres, le cristal entre l'eau et les diamants, le Mercure entre l'eau et les métaux, la marcassite entre les métaux et les pierres, le corail entre les plantes et les pierres, le Zoophyte ou Plantanimal entre les plantes et les animaux, tels que sont le Iogue des Indiens, les Nacres, les éponges et les huîtres ${ }^{2}$ ». Mersenne cite aussi « les arbrisseaux » qui « sont entre les herbes et les arbres, l'amphibie, tel qu'est le veau marin, et la loutre, est entre les poissons et les animaux terrestres: l'hermaphrodite, ou Androgyne entre les deux sexes: les poissons volants sans plume, ou qui ne volent point encore qu'ils aient des plumes, sont entre les poissons et les oiseaux, la chauve-souris, qui vole sans plumes, est entre les oiseaux et les animaux terrestres ». Évidemment, le P. Mersenne n'établit ici aucun rapport avec les jésuites, et il ne fait allusion à aucune fable.

9 En fait, deux animaux sont assez ambigus pour répondre au texte de Pascal, la chauvesouris, qui est un composé d'oiseau et de souris, et le poisson volant: comme ce dernier est le seul à tenir le milieu entre les poissons et les oiseaux, c'est évidemment lui qu'il faut retenir.

10 Comme modèle de duplicité morale, c'est surtout la chauve-souris qui a servi aux fabulistes. Ésope a composé une fable intitulée De la chauve-souris et de la belette, dans laquelle une chauve-souris, tombée à terre et attrapée par une belette ennemie des volatiles, déclare qu'elle n'est pas un oiseau, mais une souris ; et par la suite, tombée sur une seconde belette, myophobe celle-là, elle se sort d'affaire en déclarant qu'elle est une chauve-souris, et non un rat. Mais la fable ne traite pas de l'hypocrisie: elle montre seulement que l'on ne doit pas se tenir aux mêmes moyens, car ce sont ceux qui savent se transformer selon les circonstances qui échappent souvent aux dangers.

11 La fable que La Fontaine en tira (II, 5) insiste plus nettement sur l'idée de la nécessité de la duplicité dans certaines situations de danger mortel. La chauve-souris nie d'abord être une souris :

Je suis Oiseau : voyez mes ailes ;

Vive la gent qui fend les airs!

Par la suite, elle nie être un volatile :

Moi pour telle passer ! vous n'y regardez pas :

Qui fait l'oiseau? C'est le plumage.

Je suis Souris : vivent les Rats ;

Jupiter confonde les Chats.

La vraie morale, dirait Pascal, se moque parfois de la morale :

Par cette adroite repartie

Elle sauva deux fois sa vie.

Plusieurs se sont trouvés, qui d'écharpe changeants,

Aux dangers, ainsi qu'elle, ont souvent fait la figue.

Le sage dit, selon les gens :

Vive le Roi ! vive la Ligue. 
Figure 2

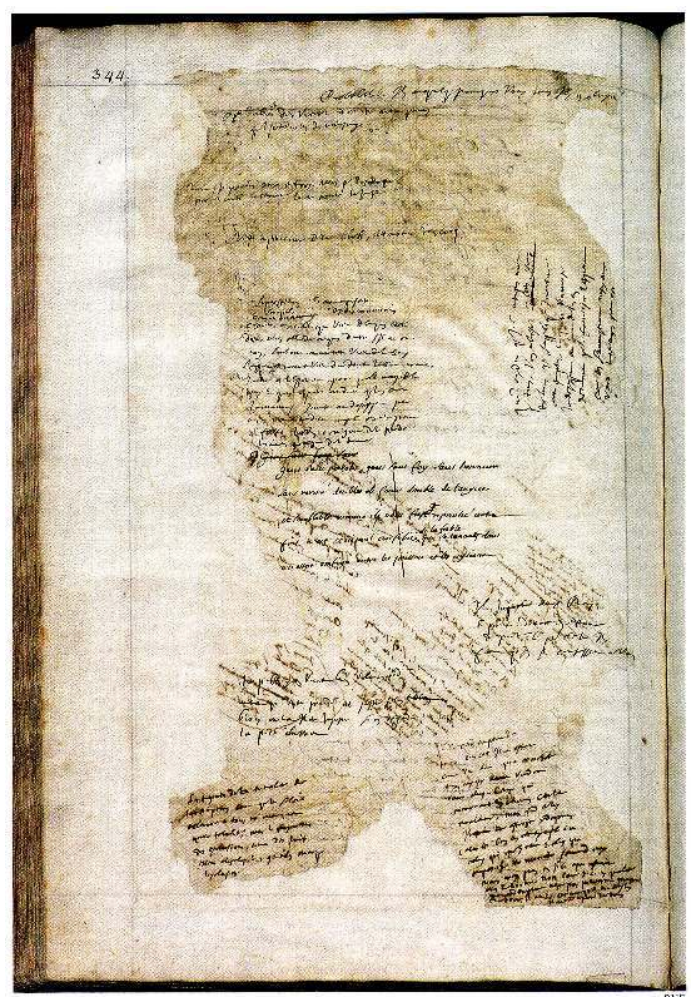

12 Cependant, la chauve-souris ne nous est d'aucun secours. Elle peut difficilement se substituer au poisson volant. On est loin, de toute façon, du sens de Pascal. La référence n'a d'intérêt que si l'on remarque que ces bêtes sont dans la fable en danger de mort, donc en situation de légitime défense, ce qui n'est nullement le cas des animaux amphibies auxquels pense Pascal, comme on va le voir.

13 Venons donc aux poissons volants. L'hirondelle de mer (arundo marina) est mentionnée chez de nombreux auteurs, mais on trouve peu de textes qui tirent de ces animaux une leçon de morale consistante. Ambroise Paré, traitant Des monstres, renvoie à Jean de Léry : il confirme avoir vu de "grosses troupes de poissons (tout ainsi que sur terre on voit les alouettes ou étourneaux) volant presque aussi haut hors de l'eau qu'une pique, quelquefois près de cent pas loin ${ }^{3}$ ". "Il est souvent advenu » que ces pauvres bêtes "se heurtant contre les mâts de nos navires, tombant dedans, nous les prenions à la main ». Le seul rapport avec la fable d'Ésope consiste en ce que Paré note, après Jean de Léry, que ces poissons volent surtout pour échapper aux gros poissons prédateurs qui leur font « une continuelle guerre »; et encore, quand ils leur échappent, ils tombent sur « certains oiseaux marins qui les prennent et s'en repaissent». A priori, les poissons volants se prêteraient assez bien à une morale analogue à celle d'Ésope et de La Fontaine. 
Figure 3

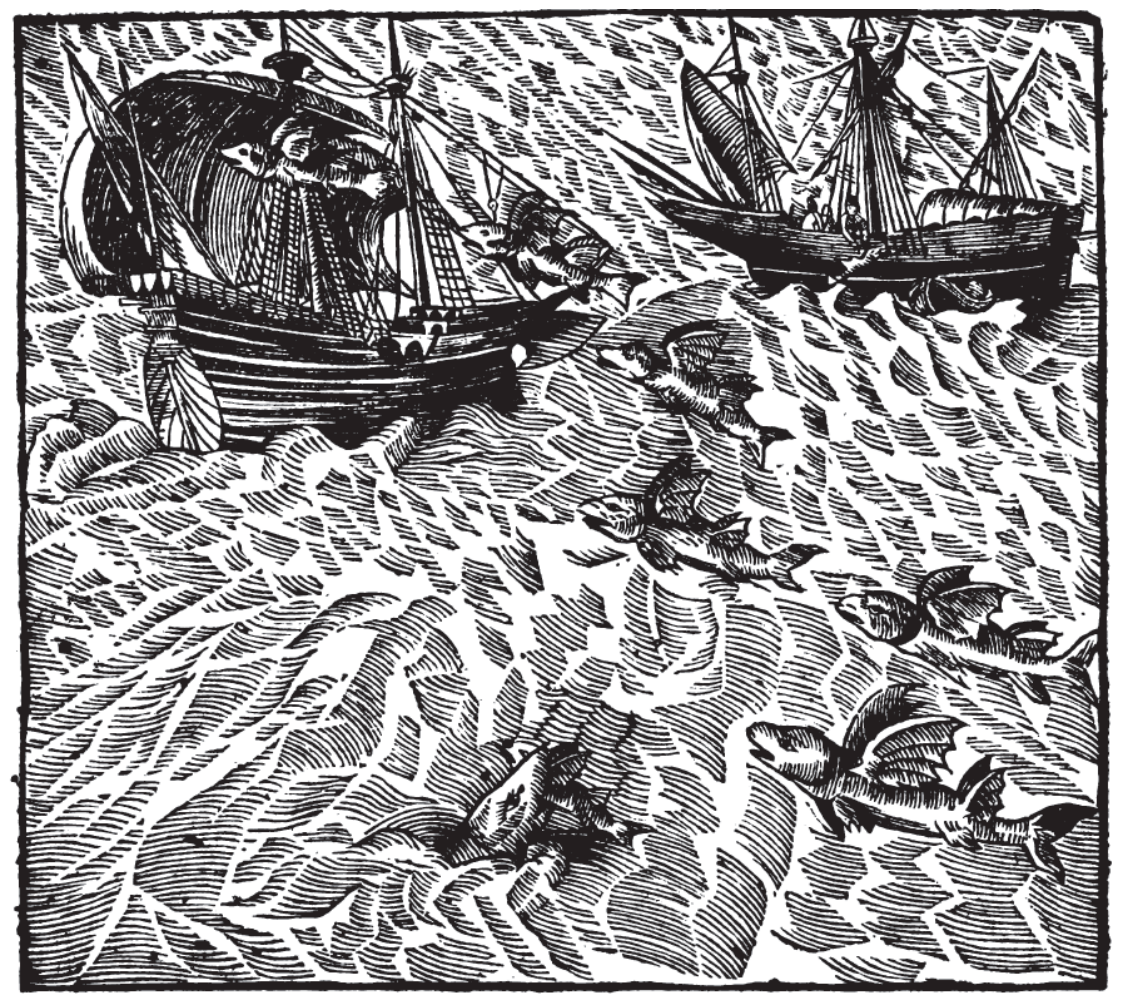

Boaistuau, dans les Histoires prodigieuses extraites de plusieurs fameux auteurs, présente l'Arondelle de mer: "Entre les prodiges de la mer, il semble miraculeux et presque incroyable que les poissons volent, et que ces animaux stupides s'élèvent de leur élément humide pour fendre et pénétrer l'air, et imiter les oiseaux, et néanmoins il est tout certain (comme on voit par expérience en plusieurs endroits de la mer) qu'il y a plusieurs "espèces de poissons volants ${ }^{4}$ ». L'auteur se contente d'en donner la figure, et de remarquer que ce poisson, par sa façon de voler, ressemble plus à une hirondelle (arondelle) qu'à une chauve-souris. 


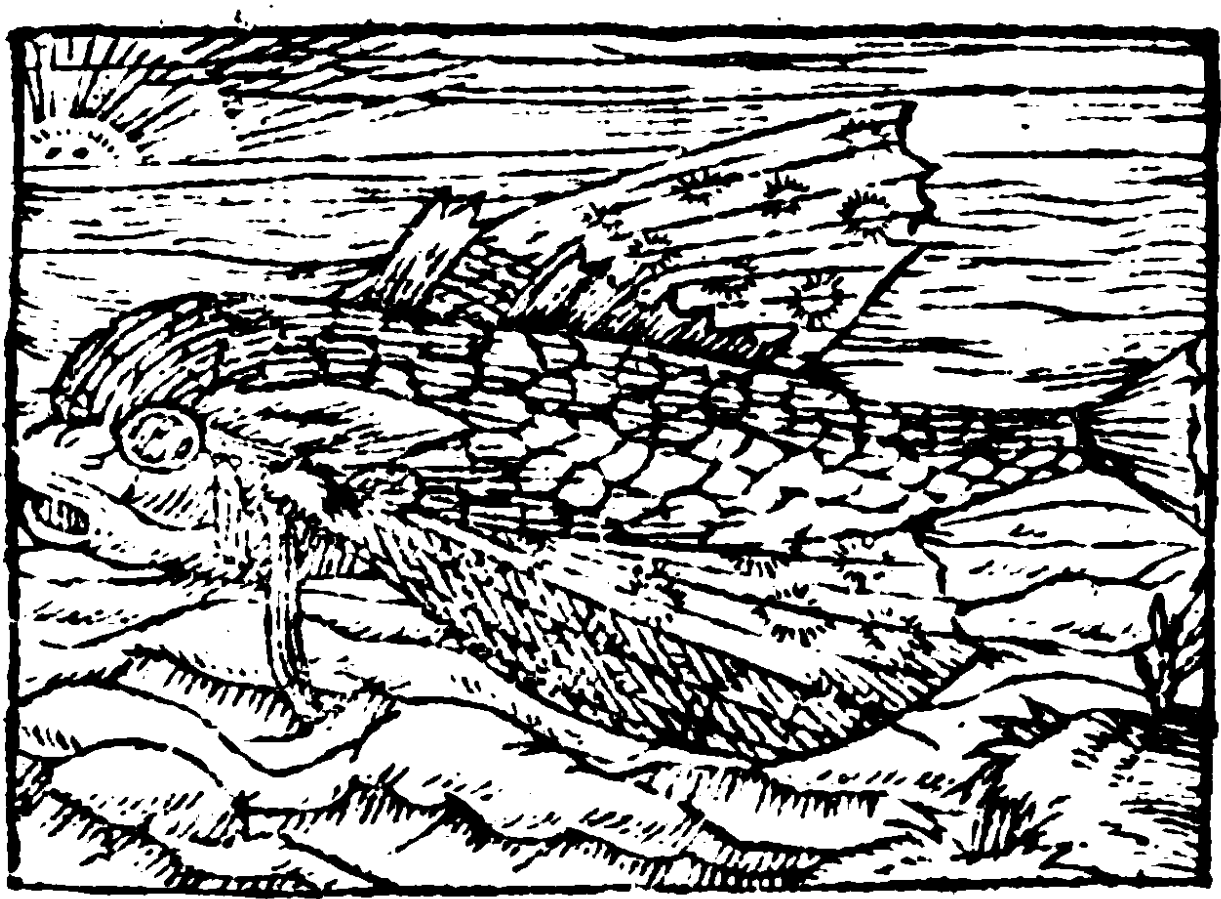

Guillaume Rondelet décrit l'arondelle de mer ou ratepenade ${ }^{5}$. Il remarque ailleurs que ce dernier nom vient de ce «que de la couleur, de la grandeur et des taches des ailes elle ressemble à un chauve-souris ou ratepenade ${ }^{6}$ ». Il parle à part de mugile alato.

Figure 5. De hirundine

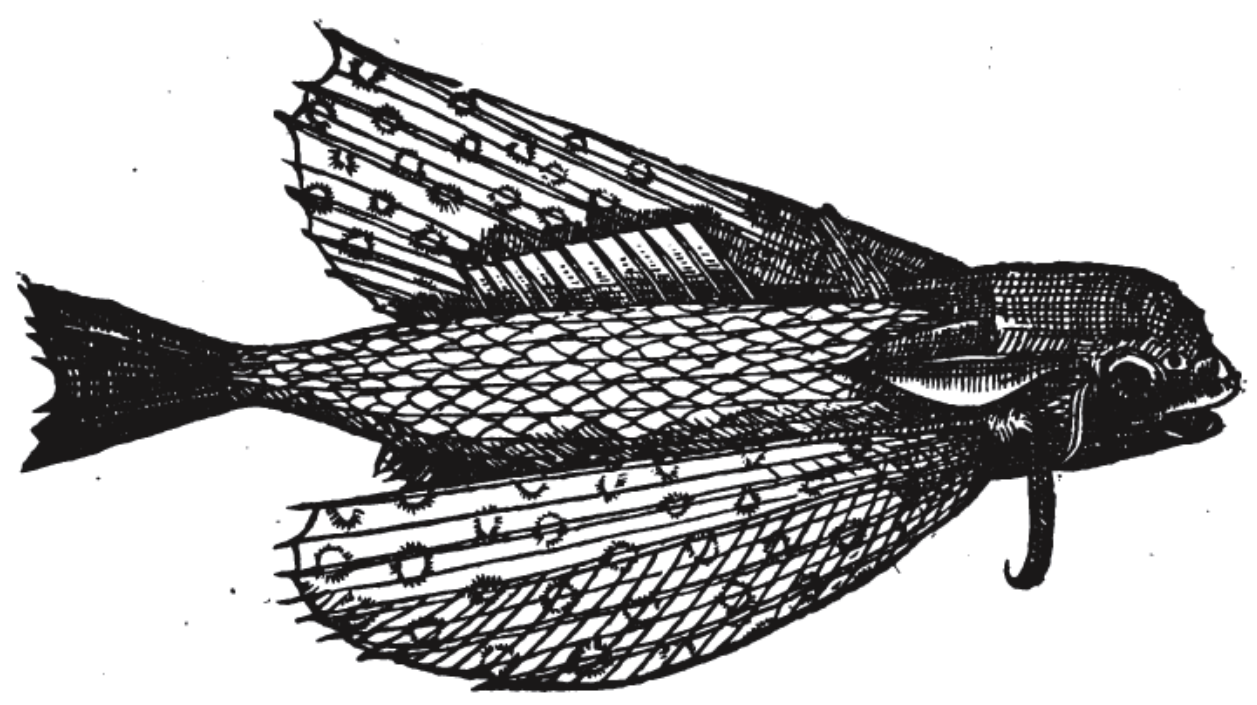




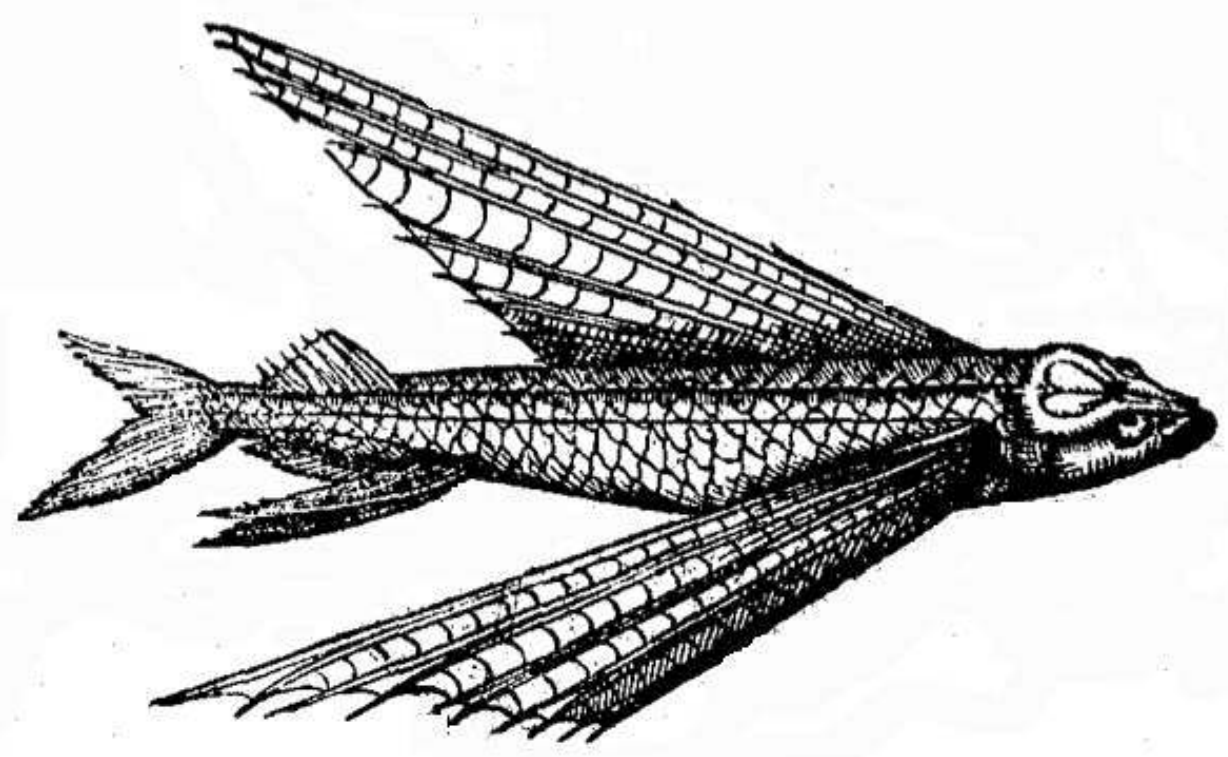

Pierre Bellon, dans La nature et diversité des poissons, présente l'hirondelle de mer en ces termes $^{7}:$ «L'hirondelle a quatre grandes ailes, sans aucun aiguillon, desquelles se sachant aider en l'air, vole quelque peu à la manière des hirondelles, dont elle en a pris son nom grec, latin et français. Sa tête est quasi aussi grande, comme est celle du mulet. Ses yeux sont grands et larges. Sa bouche petite et sans dents. Le dessus de sa tête est plat. Il n'y a qu'une pinnule ou aile sur son dos, joignant sa queue ». La queue est « fourchée et large, en laquelle chacun peut observer cette enseigne particulière ", que la "fourchure d'en bas » se trouve " plus grande que celle de dessus. Il n'y a aucun autre poisson qui ait les ailes des côtés si longues: car elles surpassent outre la queue, lesquelles avec toutes les autres tirent sur le noir. Elle nous est rare et peu connue. Et n'était qu'on la garde par singularité, lui voyant des ailes si grandes elle ne serait si fréquente : mais pour le miracle en nature on la pend avec ce qu'on garde ès cabinets entre les rares singularités ».

Figure 7

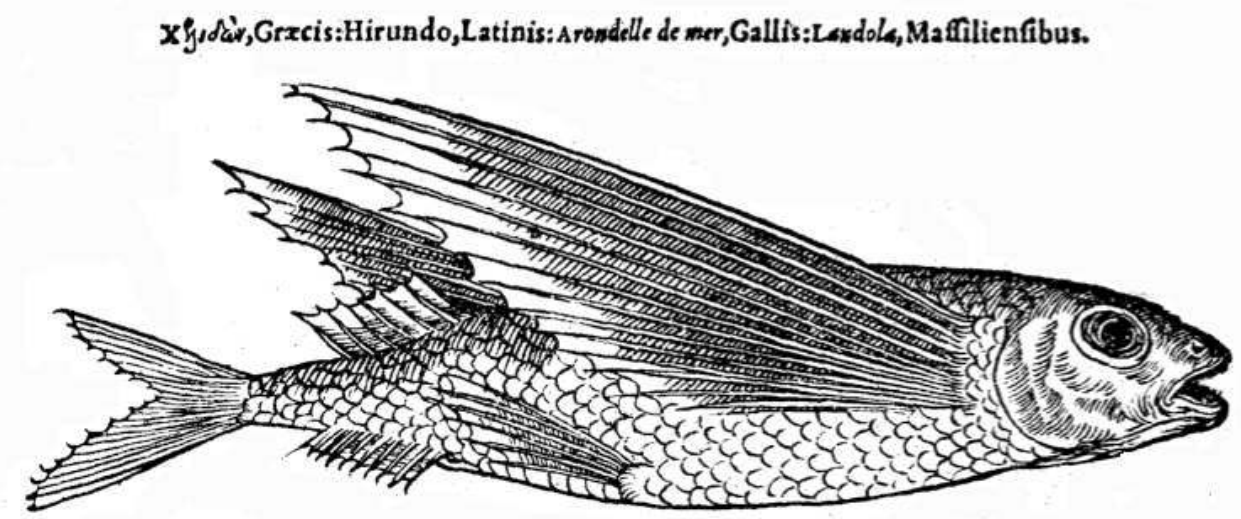

17 Une présentation très étoffée de l'arondelle de mer est donnée par Ulysse Aldrovandi, De piscibus libri $V$ et de cetis liber unus, Bononiae, ap. N. Thebaldinum, 1638. Aldrovandi 
remonte à ce que les anciens disent de cet étrange poisson, et relève les erreurs d'identification faites par ses collègues Rondelet et Bellon.

De toutes ces descriptions, on ne trouve rien qui relève d'une interprétation morale de l'animal en question, ni de la fable.

Ce n'est en fait pas du côté des spécialistes de la zoologie qu'il faut chercher.

En réalité, Pascal s'inspire d'une affaire d'usurpation des abbayes bénédictines par les jésuites, analogue à celle de Voltigerod, dont il est question sur la même page $344 \mathrm{du}$ Recueil des originaux. Il tire sans doute son information d'un ouvrage que l'on attribue à Arnauld, L'innocence et la vérité défendues contre le P. Brisacier, $1652^{8}$, écrit à l'occasion de l'affaire Callaghan, et qui cite des exemples d'exactions des jésuites sur les monastères.

21 L'ouvrage consacre de longues pages à la réfutation des calomnies dont les jésuites accablent les religieuses de Port-Royal, et en général aux usurpations par lesquelles ils parviennent à mettre la main sur des couvents pour subvenir aux besoins financiers de leurs collèges. L'avidité et la cupidité des jésuites y sont mises en forte lumière. Le livre cite « un mémoire très fidèle et très exact, qui nous a été mis entre les mains par des personnes pieuses et très instruites dans ces matières, d'une partie des abbayes et des prieurés que vous [sc. les jésuites] avez enlevés par vos intrigues, tant à l'ordre de saint Benoît, que de Cîteaux et des chanoines réguliers de saint Augustin, de la plupart desquels vous avez exterminé les moines rentés, et avez retenu les revenus qui en dépendent, pour vous enrôler parfaitement, selon vos termes, dans la confrérie du cardinal de Châtillon. Car si ce cardinal a joui de quelques abbayes et prieurés, et en a chassé les moines, il ne vous a précédés que du temps ${ }^{9} »$.

22 Le mémoire passe alors à ce second point: "Lorsqu'ils possèdent ces abbayes ou ces prieurés sous quelque charge, il n'y a point de moyens qu'ils n'emploient pour retenir les revenus, sans s'acquitter de ces charges, quoiqu'ils y soient obligés par des contrats solennels. En voici entre autres un exemple très célèbre. Nous avons déjà dit que leur collège de Rennes possède deux prieurés conventuels, dépendants de l'abbaye de $\mathrm{S}$. Florent de Saumur, qui valent ensemble sept mille livres de rente (sans un troisième qui en vaut trois mille, et qui dépend d'une autre abbaye du même ordre de S. Benoît). Lorsqu'ils entrèrent dans ces prieurés en 1606, il y eut contrat passé entre eux et les religieux bénédictins de l'abbaye de Saint Florent, qui se départirent en leur faveur de tous les droits qu'ils avaient sur ces deux prieurés, à condition qu'ils logeraient, nourriraient et instruiraient dans leur collège de Rennes, deux religieux écoliers de cette abbaye. Ils n'ont pas pu d'abord s'en dispenser; mais après que les réformés sont entrés en cette abbaye, ils ont cru en 1647 pouvoir profiter de ce changement; de sorte qu'ils refusèrent deux jeunes novices, qu'on leur avait présentés, sous prétexte que n'étant pas profès, quoi qu'on leur justifiât qu'ils en avaient auparavant reçu des novices: et ayant perdu la cause aux Requêtes du Parlement de Bretagne avec dépens, ils en appelèrent à la Cour, où sur ce que les bénédictins leur reprochaient leur ingratitude, de ne vouloir pas seulement nourrir deux jeunes écoliers pour sept mille livres de rente, ils soutinrent formellement dans des contredits écrits de la propre main d'un jésuite, qu'ils n'avaient nulle obligation à l'ordre de saint Benoit, mais aux seuls nobles bourgeois de Rennes. Sur quoi M. Denoual, avocat des bénédictins, représenta à la Cour en pleine audience, par son plaidoyer que nous avons entre les mains : «Qu'en ce seul royaume on leur pouvait coter plus de cent mille livres de rentes qu'ils possèdent du patrimoine de S. Benoît, et supplia la Cour de se souvenir que l'année précédente les mêmes jésuites plaidant contre les habitants de Rennes, avaient soutenu publiquement en la même chambre, par la bouche du même avocat, qu'ils ne leur 
avaient aucune obligation; mais bien à l'ordre de saint Benoît, duquel seul ils reconnaissaient tenir le meilleur et le plus liquide de leurs biens : ajoutant agréablement, que ces bons pères ressemblaient à cet animal amphibie de la fable, qui étant sommé de rendre ses hommages au Dauphin, roi des poissons, s'en excusa disant qu'il était oiseau ; et puis se voyant pressé de les rendre à l'Aigle reine des oiseaux, déclara qu'il était poisson ". Les suites ne sont pas à l'honneur des jésuites.

"Ainsi cette procédure ayant paru également injuste et honteuse, le Parlement confirma, par son arrêt du 7 avril 1648, la sentence des Requêtes. Mais ils en appelèrent à euxmêmes, et à leur opiniâtreté inflexible, lorsqu'ils se sont engagés dans quelque injustice : car ils logèrent et traitèrent si mal ces deux novices, que les faisant presque languir de faim et de froid, ne leur donnant aucun livre pour étudier, comme ils y étaient obligés, et les tenant sous la clef comme des prisonniers, les bénédictins furent contraints d'y faire faire une descente par un conseiller de la Cour, nommé M. Couturier, qui marque toutes ces circonstances dans son procès-verbal, que nous avons vu, n'en ayant voulu croire que nos propres yeux. Et nonobstant toutes ces poursuites, il ne fut pas au pouvoir des bénédictins et du Parlement, de faire exécuter leur arrêt. De sorte qu'à la fin ces religieux ont été contraints de retirer leurs novices, qui ne pouvaient plus souffrir un si mauvais traitement, et de quitter leur droit, pour ce qu'ils ont pu tirer de si bon amis des religieux lorsqu'ils sont obligés de les nourrir, et de si bons payeurs de leurs dettes ".

Ce passage a dû paraître mémorable : il a servi par la suite dans les polémiques contre les jésuites. Il est reproduit dans un ouvrage intitulé Les jésuites marchands, usuriers usurpateurs, et leurs cruautés dans l'ancien et le nouveau continent, Pour servir de suite au livre intitulé Les Jésuites criminels de lèse-majesté, La Haye, chez les frères Vaillant, 1759. Il est aussi cité en note dans les Annales de la Société des soi-disant jésuites, ou Recueil historiquechronologique des tous les actes, écrits, dénonciations, avis doctrinaux, requêtes, ordonnances, mandements, instructions pastorales, décrets, censures, bulles, brefs, édits, arrêts, sentences et jugements émanés des tribunaux ecclésiastiques et séculiers, contre la doctrine, l'enseignement, les entreprises et les forfaits des soi-disant jésuites, depuis 1552, époque de leur naissance en France, jusqu'en 1763, tome IV, Paris, 1769, p. 675-676.

26 Il a donc sans doute existé une autre version de la fable, consacrée aux poissons volants, qui permit aux défenseurs des bénédictins de placer un trait plaisant dans une cause sinistre d'usurpation, de spoliation et de séquestration, dans laquelle les jésuites tiennent évidemment un vilain rôle.

Le sens de cette allusion s'éclaire du fait que ce passage est situé, dans L'innocence et la vérité défendues, tout proche du récit des exactions des jésuites à l'égard des religieuses de Voltigerod, auxquelles Pascal fait allusion dans le fragment que nous examinons.

«Le Port-Royal», écrit Pascal, "vaut bien Voltigerod». Cette fois, la source a été reconnue par les éditeurs des Pensées.

Figure 8

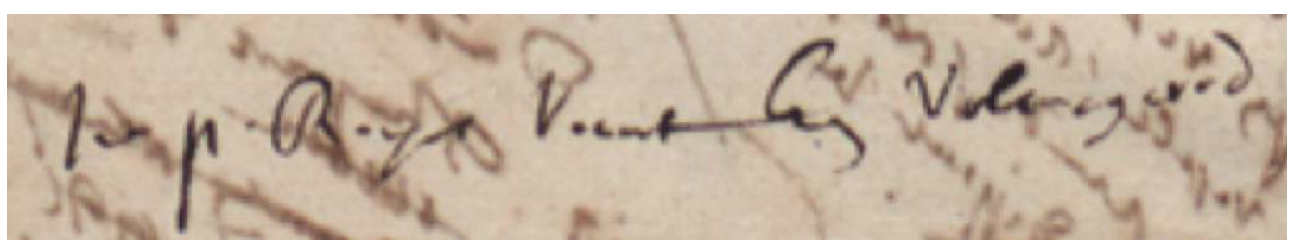

Courrier du Centre international Blaise Pascal, 34 | 2015 

Grolar, en Basse-Saxe. En 1631, les Jésuites ont tenté de s'emparer de cette abbaye, en faisant expulser les religieuses cisterciennes qui l'occupaient. Pascal comptait utiliser un dossier constitué par le groupe de Port-Royal sur les tentatives faites par les Jésuites pour s'emparer de plusieurs abbayes bénédictines. Ce dossier devait comprendre un factum de Dom Vuillaume sur les affaires d'Alsace ${ }^{10}$ et des ouvrages du bénédictin allemand Dom Hay, Astrum inextinctum (1636), et Hortus crusianus (1648). L'Astrum inextinctum a servi à Arnauld dans L'Innocence et la vérité défendues. Il n'a pas donné suite à son projet, et seule une allusion obscure subsiste dans la XVI ${ }^{e}$ Provinciale: «Ne semble-t-il pas qu'on ne peut convaincre d'imposture une reproche si indéterminé ? Un habile homme néanmoins en a trouvé le secret; et c'est encore un Capucin, mes Pères. Vous êtes aujourd'hui malheureux en Capucins, et je prévois qu'une autre fois vous le pourriez bien être en Bénédictins ${ }^{11}$ ». Le dossier a été utilisé plus tard par Pontchâteau dans la Morale pratique des Jésuites ${ }^{12}$.

Dans La vérité et l'innocence défendues, Arnauld soutient que, si les jésuites avaient pu obtenir la dispersion des religieuses de Port-Royal, ils auraient aussitôt trouvé moyen de profiter de la situation pour s'emparer de l'abbaye pour en user à leur profit. «Vous auriez eu moins d'aversion pour les édifices que pour les personnes; et vous vous seriez aisément persuadés qu'il n'aurait point fallu d'autre feu que celui de votre bouillante charité, pour purifier le mauvais air de la contagion du Cyranisme, ainsi que vous l'appelez. Vous n'auriez point manqué d'arguments pour inspirer à ceux qui vous auraient voulu croire, qu'on ne pouvait, sans se rendre coupable de la perte et de la damnation de plusieurs âmes, refuser à votre société ce moyen si innocent de s'accroître et de s'agrandir, et de travailler plus puissamment à la conversion des infidèles et des hérétiques ${ }^{13}$ ». Arnauld note pour conclure : «On peut donc apprendre par ces exemples ce que les filles de Port-Royal auraient dû attendre des jésuites, si le pouvoir de ces bons religieux avait été égal à leur animosité. Car s'ils ont traité d'une manière si inhumaine ces religieuses, qu'ils n'avaient aucun sujet de persécuter, sinon parce qu'elles avaient du bien, qui pouvait être commodément employé à enrichir leurs collèges, que n'auraient-ils point fait contre celles qui, depuis tant d'années, font l'objet continuel de leur passion et de leurs injures ${ }^{14}$ ?».

31 Cette dernière formule est donnée sous forme plus lapidaire par Pascal : Port-Royal vaut bien Voltigerod, Port-Royal est aussi alléchant pour les jésuites que Voltigerod.

Les poissons volants ne sont au fond qu'un trait plaisant d'avocat, qui n'a pas survécu à cette note du Recueil des originaux. Mais les épisodes auxquels Pascal fait allusion figurent parmi les plus sinistres exactions que les jésuites aient commises. Et si l'on pense que, dans l'esprit de Pascal, ce qui était arrivé à Voltigerod pouvait bien arriver au monastère où vivait sa sœur Jacqueline, on comprend que la plaisanterie ait chez lui laissé place aux accents puissants de la XVI ${ }^{\mathrm{e}}$ Provinciale. 


\section{ANNEXES}

\section{Relecture de La Morale pratique des Jésuites de Pontchâteau}

"Mensonges et fourberies des jésuites pour s'emparer d'une abbaye de religieuses bernardines en Saxe. Nous venons de voir dans l'histoire précédente que l'empereur Ferdinand II ayant résolu de tirer d'entre les mains des protestants les abbayes qu'ils avaient occupées depuis le traité de Passau fait en 1552 il avait ordonné par son édit public du 6 mars 1629, qu'elles seraient rendues au religieux des ordres auxquels elles appartenaient par leur première fondation. L'abbé du monastère de Cesarée de l'ordre de Cîteaux étant député par son général pour travailler à l'exécution de cet édit de l'empereur envoya l'abbé de Valenciennes, qui mena avec soi quatre religieuses professes bernardines, accompagnées de deux novices et d'une scur converse, pour mettre en possession de l'abbaye de VOLTIGERODE dans la Basse Saxe. Et l'évêque d'Osnabrug, l'un des commissaires de l'empereur, les y ayant établies par l'un de ses officiers, elles y demeurèrent plusieurs mois, $y$ faisant le service divin, et tous les autres exercices de la vie religieuse.

Mais les jésuites ayant dessein d'enlever cette abbaye, aussi bien que toutes celles des religieuses que les hérétiques devaient rendre, employèrent auprès de l'empereur le crédit de leur père Lamorman, qui se servit de deux insignes mensonges pour se la faire donner. Le premier fut que les abbés députés des ordres de S. Benoît et de Cisteaux leur avaient cédé volontairement toutes les abbayes de filles, et quelques-unes d'hommes des moins célèbres. L'autre que l'abbaye de Voltigerod, qui est proche de la ville impériale de Goslar, ÉTAIT DÉ SERTE, ET QUE PERSONNE NE L'AVAIT REDEMANDÉE ; et qu'elle serait FORT COMMODE AUX PÈRES DE LA SOCIÉTÉ, qui voulaient faire un noviciat dans cette ville, où ils avaient déjà un collège. Ce qui fut exprimé en propres termes dans la commission qu'ils obtinrent. L'un et l'autre était une fausseté signalée ; puisqu'il y avait déjà plusieurs mois que les religieuses de Cîteaux étaient en possession paisible de cette abbaye.

Mais comme les démons, selon un saint docteur, prophétisent ce qu'ils veulent faire, ces bons pères travaillèrent aussitôt à rendre vrai ce qu'ils avaient dit faussement. Le premier moyen qu'ils employèrent fut la fourberie. Ayant persuadé à ces bonnes filles qu'elles n'étaient pas en sûreté dans cette abbaye de la campagne ; qu'elles étaient exposées aux courses des soldats et aux violences de la guerre; et qu'il était à propos qu'elles la quittassent pour un temps; et qu'elles se retirassent à Goslar, où ils les firent recevoir dans le monastère de Franquembeg au mois de mars 1631. Mais quoique ces religieuses, qu'ils avaient épouvantées par cet artifice, en fussent sorties, elles y laissèrent tous leurs menbles, tous leurs serviteurs, tous leurs bestiaux, et tout leur ménage.

Cette supercherie ayant si bien réussi aux jésuites, ils firent bientôt voir à ces bonnes filles, qu'il n'y avait point d'autres courses de soldats, ni d'autre violence qu'elles dussent craindre, que la leur propre. Car peu de jours après, savoir le 29 du même mois de mars, le provincial de la Compagnie, nommé Herman Gauvinz, s'empara de l'abbaye, y laissant quelques jésuites; et contraignit par force les serviteurs des religieuses qu'elles y avaient laissés, de leur faire 
serment de fidélité, sans en avoir rien signifié aux supérieurs de l'ordre de Cîteaux, ni à l'abbé de Cesarée administrateur de ce monastère.

\section{Leur cruauté inouïe à chasser par force ces filles et leur confesseur, de cette abbaye}

Mais ces filles se voyant si malicieusement trompées, trouvèrent moyen de rentrer secrètement dans leur abbaye ; et s'étant mises dans le chœur d'en-haut de l'église, elles y demeuraient nuit et jour, y célébrant tout le service divin, les jésuites occupant le reste des logements. Ce retour des filles fâcha ces bons pères. Il n'y eut rien qu'ils ne tentèrent, soit par douceur, soit par menaces, pour les en faire sortir ; et sans des paysannes hérétiques, voisines de cette abbaye, qui touchées de pitié leur apportaient du lait en cachette, ils les auraient fait mourir de faim. Mais voyant qu'elles demeuraient fermes, malgré tous ces mauvais traitements, ils résolurent de les en chasser par violence. Et ayant fait venir des sergents et des soldats le 12 d'avril veille du dimanche des Rameaux, eux étant présents, et un de leurs novices faisant le principal personnage de cette irréligieuse tragédie, par une témérité, ou plutôt par une cruauté inouie parmi des religieux, ils arrachèrent par la force de l'église même, en un temps si saint, des vierges consacrées à Dieu, les enlevèrent parmi leurs gémissements et leurs cris, avec le scandale de toute cette province. Et ce novice les traita si mal, que l'une d'elles en ressentit plus la fureur que les autres, en demeura longtemps malade.

Cette histoire est si surprenante, qu'on pourrait croire aisément que le P. Hay qui la rapporte, aurait usé de quelque exagération si ce qu'il en dit n'était encore moins que ce qui est prouvé par des actes authentiques de justice, qu'il a inséré tout au long, tant en allemand qu'en latin. Car voici le procès verbal qui en fut fait, et envoyé à l'official d'Osnabrug, où la plainte des religieuses faites en allemand, est insérée en ces termes traduits en latin.

Nous ne pouvons pas, étant de pauvres pupilles abandonnées, ne point élever notre voix, pour nous plaindre de l'état misérable où nous a réduites l'étrange et cruel procédé, que les pères jésuites ont exercé contre nous le soir du samedi veille des Rameaux. Car étant venus avec le seigneur Widelag et deux sergents, qui sont les ministres ordinaires dont les magistrats se servent pour prendre les voleurs et les scélérats, dans notre abbaye de Voltigerod, dans laquelle nous avions été établies par notre père spirituel l'abbé de Walhenriedh commissaire subdélégué, conformément à l'édit de restitution de sa Majesté impériale, ils arrivèrent entre six et sept heures, et nous trouvant dans le chœur de l'église où nous faisions nos prières, ce seigneur et les jésuites nous parlèrent fièrement et nous pressèrent de sortir. Mais nous demeurâmes à genoux dans nos chaires, et nous répondîmes que nous étions sous l'obéissance de notre saint ordre, et qu'il ne nous était pas permis de sortir de notre maison sans le commandement de son supérieur. Après cela moi religieuse professe MARIE KOGEL, prenant nos sièges avec mes deux mains, je m'y attachai de toutes mes forces : mais ces deux sergents et le jésuite novice m'arrachant les mains par force, me prirent et le jésuite me tint serrée de ses deux bras par le milieu du corps; et ainsi ils me portèrent en partie, et en partie me traînèrent jusqu'au bout du chœur ; et comme je criais VIOLENCE, JÉSUS VIOLENCE. JE CROIS QUE VOUS ME TUEREZ (car je ne pouvais plus respirer) ils me tirèrent hors du chœur. Et ce fut là que notre confesseur étant arrivé, me trouva couchée par terre, qui m'écriais contre la violence qu'on me faisait : mais m'ayant fait relever de force, ils me mirent dans une chaire pour m'emporter, comme ils firent, me faisant faire tout le tour de l'abbaye, et enfin me jetèrent hors de la cloture, où marchant au milieu d'un champ étant entre ces deux sergents, je fus menée par les bras comme une larronnesse et une méchante. Et en allant ayant rencontré un chariot, je me jetai à la roue, d'où ils m'arrachèrent avec tant de violence, que le lendemain je me trouvai toute meurtrie par les bras, 
et sentant une si grande douleur dans la poitrine (sans parler de la frayeur et de l'émotion que cette violence m'a causée) que je ne sais pas si j'en relèverai jamais.

Après moi suivit la noble vierge ANNE LUCIE DERNBACH, proche parente du vice-chancelier de sa majesté impériale, laquelle ils enlevèrent de la même sorte, et avec la même violence, en présence de notre confesseur, qui eut beau reprocher au père recteur des jésuites, qu'il n'aurait jamais cru qu'il fût capable de faire jouer une telle tragédie en un temps si sacré, et eut beau représenter à ces sergents qu'ils se souvinssent que c'était la parente de Monsieur de Stralendorf vice chancelier de l'empire qu'ils traitaient de la sorte, il ne gagna rien par ses remontrances, mais on l'enleva comme on m'avait fait.

La troisième, propre sœur de la précédente, était ANNE SIDONIE DE DERNBACH, à laquelle ils arrachèrent les mains des chaires du chœur avec la même violence, et le jésuite novice la serrant de ses deux bras par le milieu du corps, l'entraîna dehors, et la mit sur une chaire pour l'emporter : pendant quoi elle criait au jésuite, si c'était là leur reconnaissance des grands biens que son cousin avait faits à leur collège de Fulde : que cette injure retomberait sur monsieur le vice chancelier de l'Empire. Mais elle parlait à des sourds. Ils firent le même traitement à deux autres religieuses. Et nous pouvons assurer devant Dieu, et devant toute la cour céleste, que ce que nous venons de réciter est la pure vérité.

On ne peut entendre le récit d'une histoire si pitoyable, sans être également touché de compassion envers des religieuses de piété, et d'une naissance illustre, si cruellement traitées dans leur propre monastère ; et d'indignation contre les auteurs d'une si barbare violence. Mais elle paraîtra encore plus honteuse, lorsque nous y aurons ajouté quelques autres circonstances, qui sont très fidèlement rapportées par le P. Hay, célèbre religieux bénédictin, en ces propres termes. Autrefois, dit-il, dans la vieille loi, les criminels qui s'enfuyaient dans le temple, lequel n'était purifié que par le sang des boucs et des veaux, trouvaient leur sûreté dans cet asile, s'ils pouvaient prendre la corne de l'autel. Et aujourd'hui dans la loi nouvelle, les pères de la société ne font point de conscience de se servir de sergents et des ministres des bourreaux, pour s'emparer avec insolence des temples dédiés au Dieu vivant, consacrés par les redoutables mystères de JÉSUS-CHRIST, et d'en arracher par force d'innocentes religieuses. Quelle honte! Quelle infamie! Le révérend père David, prieur des dominicains d'Alberstad, et un frère convers nommé Ange, se trouvèrent par rencontre à un spectacle si triste, et si inouï: et ils reprochèrent avec tant de zèle et tant de chaleur à ces jésuites l'énormité de cette action qu'il s'en fallut peu que le frère convers ne se mît en devoir de les repousser. Mais les jésuites ne se contentèrent pas de la violence qu'ils avaient faite aux religieuses, ils crurent qu'il leur était encore nécessaire de chasser par force de cette même abbaye leur supérieur et confesseur, qui étant un religieux de l'abbaye de Cesarée, nommé le père Michel Gorz. Il revenait par rencontre de la ville de Brunsvic, où il était allé requérir des calices appartenant à cette abbaye de filles. Et étant retourné assez tôt pour être le spectateur de cette tragédie, il en fut le dernier acteur. Car ayant reproché en face à ce recteur des jésuites l'indignité de l'outrage qu'il faisait à ces saintes vierges, parce qu'il ne voulait pas s'en aller, selon que ces pères le lui commandaient, et qu'il s'était retiré dans le cimetière, comme pour y chercher quelque sûreté parmi les morts, on ordonna à deux soldats, qui faisaient difficulté de mettre les mains sur ce prêtre, de jeter aux dés à qui le chasserait dehors. Cela ayant été fait, l'un d'eux le prit, et le jeta violemment hors de la porte du monastère. Ce qui obligea un soldat protestant de Mechelbourg, touché de ce lamentable spectacle, de dire avec indignation aux jésuites: On ne souffrirait pas en notre pays qu'on traitât ainsi nos ministres. Voilà quelle fut la fin de L'ÉTABLISSEMENT CANONIQUE (comme l'appelle le jésuite Crusius) des pères de cette société dans l'abbaye de Voltigerode. 


\section{Un abbé de l'ordre de Cîteaux les en fait sortir honteusement, et y rétablit les religieuses}

Mais l'abbé de Cesarée administrateur de cette abbaye, qui avec l'autorité de l'empereur y avait rétabli les filles selon l'édit, ayant eu avis de cet horrible procédé, en écrivit en ces termes au P. Lamorman jésuite, confesseur du même prince : J'ai appris des choses qui sont pour moi tristes et funestes : l'événement apprendra avec le temps si elles seront utiles et avantageuses pour ceux qui n'y ont regardé que leur profit et leur avantage. Vous avez joué, mes Pères, un jeu bien étrange dont je vous envoie la relation, qui s'étant rencontré dans le temps de la Passion de JÉSUS-CHRIST, nous en a malheureusement représenté l'image et la forme. Mais il s'est trouvé deux différences bien étonnantes. L'une que ç'ont été des filles qui ont représenté la personne de JÉSUS-CHRIST ; l'autre que ceux qui prennent le nom de JÉSUS, étant accompagnés de leurs satellites, n'en ont pas joué le personnage : mais plutôt celui des Juifs qui ont persécuté et entraîné ce Sauveur. O société de Jésus ! Est-ce là la société que vous avez avec JÉSUS ?Je conjure votre paternelle révérence par les entrailles de la miséricorde du rédempteur, qu'elle fasse rendre les abbayes dont sa société s'est emparée sous le prétexte d'une FAUSSE CESSION, de peur que les anges de paix, selon le langage de l'Écriture, ne continuent toujours leurs gémissements et leurs larmes. Que si on ne fait pas cette restitution, nous ne manquerons pas de moyens pour la faire faire. À Cesarée le 30 de mai 1631.

Et en effet quelque pouvoir qu'eût ce jésuite sur l'esprit de l'empereur, l'ordre de Cîteaux ayant poursuivi près de sa majesté impériale le rétablissement de ces filles dans leur abbaye, il l'obtint par un arrêt solennel, et les jésuites furent obligés d'en déloger honteusement, comme n'y étant entrés que par une intrusion violente contre tout droit civil et canonique, et pour laquelle selon les canons ils méritaient d'être exemplairement châtiés. Car les anciens religieux de S. Benoît, de S. Bernard, et les autres, n'avaient besoin que de l'autorité de l'empereur, pour être rétablis dans leurs propres abbayes, parce que c'était leur bien que les hérétiques leur avaient ravis par violence, et dans lequel ils rentraient naturellement. Mais outre que le don de cette abbaye que les jésuites prétendaient leur avoir été fait par l'empereur, était nul en soi, pour être contraire à l'édit, et n'avoir été obtenu que par une manifeste surprise, comme il a déjà été remarqué, les jésuites euxmêmes reconnaissaient par leurs livres, qu'il n'y avait que le pape qui pût faire ces translations d'abbayes des anciens ordres à leur compagnie. Et cependant quand on les pressait de montrer que le pape leur eût donné celle-ci par quelque rescrit ou quelque bulle, n'en ayant aucun, ils répondaient par une illusion digne d'eux : Que LE PAPE la leur avait donnée, PAR L'EMPEREUR : comme si, dit le P. Hay, le pape avait accoutumé d'accorder ces grâces extraordinaires par des commissions séculières des empereurs, ou des rois, et non par des bulles ou des brefs apostoliques.

Les jésuites ne laissèrent pas depuis de se vouloir emparer de diverses abbayes sous le spécieux prétexte de la plus grande gloire de Dieu : mais la noblesse catholique du Rhin en Weteravie, se sentit obligée d'en faire des plaintes publiques au pape Urbain VIII où ils se plaignent hautement de leur avarice. Nous voyons, disent-ils, très saint Père, non sans grand étonnement, que les pères de la société de Jésus par diverses persuasions et flatteries envers les souverains chefs et princes de l'empire, outre leurs grandes richesses, se veulent encore emparer des abbayes, des fondations, et des monastères, principalement de ceux des vierges nobles et illustres, sous divers prétextes de propagation de la foi, et de l'avancement du salut des âmes. Ils représentaient ensuite : Que dans ces lieux saints que les jésuites occupaient, on n'y voyait plus aucune trace des anciennes fondations, ni des œuvres de miséricorde et de charité qui s'y faisaient 
auparavant ; que les monastères étant abandonnés, périssaient peu à peu contre les pieuses intentions de leurs ancêtres; que les bâtiments en tombaient par terre ; et qu'il n'y avait que les biens et les revenus qui en demeurassent pour enrichir les collèges des jésuites des dépouilles des anciens ordres.

Ainsi quelque vanité que des pères se donnaient, et quelque mépris qu'ils fissent des monastères de religieuses, en disant Que la virginité des filles consacrées à Jésus-Christ est une virginité solitaire, recluse, oisive, qui ne travaille que pour son salut particulier; au lieu que la leur est publique, agissant, prêchante, ardente du zèle des âmes, leur cupidité et leur avarice n'en parurent que plus odieuses, n'y ayant personne qui puisse souffrir sans indignation, qu'ils aient une si haute présomption de leur compagnie, qu'ils osent prétendre que toute la religion est en danger de tomber par terre, si on ne change en des fermes de leurs collèges, dont les dérèglements sont assez connus, les demeures saintes des vierges religieuses, dont les prières continuelles sont $s i$ utiles à l'Église et aux royaumes. »

\section{NOTES}

1. P. 79. En marge : Esa. 9. 2 Tim. 2. La seconde citation n'est qu'approximative; elle devrait finir par « ut ei placeat cui se probavit »; mais le sens correspond. En marge se trouve aussi la mention Apoc. 13, passage où il est question de la bête à sept têtes et à dix cornes « et sur ses cornes dix diadèmes et sur ses têtes des noms de blasphème. Cette bête [...] était semblable à un léopard, ses pieds étaient comme des pieds d'ours, sa gueule comme la gueule d'un lion » (tr. de Port-Royal).

2. Marin MERSENNE, La vérité des sciences, I, XI.

3. Ambroise PARÉ, CEuvres, Lyon, Rigaud, 1652, p. 685. Il renvoie à Jean de Léry, Histoire de la terre du Brésil, ch. III. Voir aussi Pline, Livre IX, ch. 27, et livre XXXII, ch. dernier.

4. Pierre BOAISTUAU, Histoires prodigieuses extraites de plusieurs fameux auteurs, Anvers, Guislain Janssens, 1594, p. 97.

5. Guillaume RONDELET, La première partie de l'histoire des poissons, Livre II, Chapitre I, Lyon, M. Bonhomme, 1558, p. 224 sq. Ratepenade est en fait le nom de la chauve-souris. Voir aussi le Livre X de son De piscibus marinis, Lyon, Mathias Bonhomme, 1554, ch. I, De hirundine, p. 284 sq.

6. Mace волномме, La première partie de l'histoire entière des poissons, Livre X des poissons, Lyon, 1558.

7. Pierre BELLON, La nature et diversité des poissons, Paris, Ch. Estienne, 1555, p. 189-193. Bellon en donne aussi une description en latin dans son De aquatilibus libri duo, 1553, Livre I, p. 193-195.

8. Antoine ARNAULD, Cuvres, XXX, p. 99 sq ; voir la notice préliminaire du t. XXX, p. VII-VIII. Sur John Callagan et les polémiques dont il a été l'objet, voir le Dictionnaire de Port-Royal.

9. Voir p. 95. Exterminer doit être entendu au sens de chasser de chez soi.

10. Voir le titre dans Arnauld, Euvres, XXXII, p. 111.

11. Provinciales, éd. Cognet, p. 291, n. 2.

12. La morale pratique des jésuites, éd. de Cologne, 1669, p. 162-170. Voir aussi Morale pratique des Jésuites, Préface, in Arnauld, CEuvres, XXXII, p. 6.

13. Antoine ARNAULD, CEuvres, XXX, p. 145. Sur Dom Hay, voir J. François, Bibliothèque générale des écrivains de l'ordre de Saint-Benoît, Bouillon, 1767, t. I, p. 452 ; ses ouvrages concernant sa polémique avec le jésuite Crusius sont à la $\mathrm{BN}$.

14. Antoine ARNAULD, L'innocence et la vérité défendues, Article III, Histoire mémorable du procédé qu'ont tenu les jésuites, pour enlever aux religieux de saint Benoît, de saint Augustin, de Cîteaux, et de 
Prémontré, les abbayes que l'empereur Ferdinand II avait retirées des protestants d'Allemagne, CEuvres, XXX, p. 148 sq.

INDEX

Mots-clés : Pascal, jésuites, hypocrite, chauve-souris, poissons volants, morale Keywords : bat, flying fish, moral 